\title{
Safety of denosumab versus zoledronic acid in patients with bone-related diseases: a systematic review and meta-analysis
}

\author{
Wenhao Luo ${ }^{1^{*}}$, Ruoyu $\mathrm{Ji}^{2^{\star}}$, Ziyao Fu², Shijie Yang ${ }^{2}$, Jing Zhan ${ }^{2}$, Ye Li ${ }^{3}$
}

\begin{abstract}
${ }^{1}$ Department of General Surgery, Peking Union Medical College Hospital, Chinese Academy of Medical Sciences and Peking Union Medical College, Beijing, China 2Peking Union Medical College Hospital, Chinese Academy of Medical Sciences and Peking Union Medical College, Beijing, China

${ }^{3}$ Department of Orthopedic Surgery, Peking Union Medical College Hospital, Chinese Academy of Medical Sciences and Peking Union Medical College, Beijing, China

*Wenhao Luo and Ruoyu Ji contributed to this article equally.
\end{abstract}

Submitted: 24 March 2021; Accepted: 16 September 2021 Online publication:

Arch Med Sci

DOI: https://doi.org/10.5114/aoms/142322

Copyright @ 2022 Termedia \& Banach

\section{Abstract}

Introduction: The aim of the study was to compare the safety of denosumab (Dmab) versus zoledronic acid (ZA) in patients with bone-related diseases. Both Dmab and ZA have been widely used in the treatment of bone-related diseases, but which drug is an optimal treatment in terms of safety remains controversial.

Material and methods: PubMed, Embase, Web of Science, the Cochrane Central Library, and ClinicalTrials.gov were systematically searched up to $1^{\text {st }}$ January 2021, and were evaluated by Preferred Reporting Items for Systematic Reviews and Meta-analysis (PRISMA) guidelines. Randomized controlled trials comparing relevant outcomes of Dmab versus ZA in patients with bone-related diseases were included.

Results: A total of 13 studies involving 21,042 participants were included. The incidence of total adverse events was significantly lower in patients receiving Dmab treatment than in those undergoing ZA treatment $(O R=0.84$, $95 \% \mathrm{Cl}: 0.75-0.94, p=0.003)$. Nine trials comparing Dmab with ZA further indicated that Dmab was significantly better than ZA in controlling the incidence of serious adverse events (OR $=0.91,95 \% \mathrm{Cl}: 0.85-0.99, p=0.02)$. Compared to ZA, Dmab administration was correlated with a lower risk of skeletal-related events $(\mathrm{OR}=0.77,95 \% \mathrm{Cl}: 0.70-0.85, p=0.00001)$. However, no significant difference was found in the rate of infection events between Dmab and ZA (OR $=1.06,95 \% \mathrm{Cl}: 0.93-1.20, p=0.39)$.

Conclusions: This study demonstrated superiority of Dmab over ZA in treating bone-related diseases in terms of safety.

Key words: denosumab, zoledronic acid, bone-related diseases, adverse events.

\section{Introduction}

With the increase of tumor incidence and the aging of the population, the prevalence of bone-related diseases along with the demand for corresponding medications is growing. We attached great importance to bone-related diseases [1-3]. As two potent antiresorptive

\author{
Corresponding author: \\ Ye Li MD \\ Department of Orthopedics \\ Surgery \\ Peking Union Medical College \\ Hospital \\ Chinese Academy of Medical \\ Sciences and Peking Union \\ Medical College \\ Post code: 510282 \\ Beijing, 100730, China \\ Phone: (86)17374509139 \\ E-mail: liye@pumch.cn
}


agents, both denosumab (Dmab) and zoledronic acid (ZA) [4] have been widely used in the treatment of bone-related diseases, including but not limited to osteoporosis $[5,6]$, bone metastases secondary to solid tumors [7-9], multiple myeloma $[10,11]$ and giant cell tumor of bone $[12,13]$. As a potent intravenous bisphosphonate, ZA plays a critical role in the prevention of skeletal complications in bone-related diseases $[5,14]$. Denosumab is a fully human monoclonal antibody of the immunoglobulin G2 isotype, which functions against the receptor activator of nuclear factor $\kappa \mathrm{B}$ ligand (RANKL) and thereby inhibits osteoclast activation and function [15], and its use is significantly less limited to renal toxicity [16]. Growing evidence suggests that Dmab is superior in terms of efficacy $[17,18]$, safety [5] and even cost-effectiveness [19, 20] over ZA. Published meta-analyses comparing the efficacy between Dmab and ZA for treatment of bone metastases in patients with solid tumors demonstrated that Dmab was better than ZA in preventing complications and delaying the onset of skeletal-related events (SREs) [21-23]. However, meta-analyses evaluating the safety between Dmab and ZA are still insufficient. In the few studies evaluating this, the use of both drugs was confined to the treatment of patients with bone metastases [16, 21, 23]. With the continuous expansion of indications of both drugs and increased interest in identifying the optimal treatment for bone-related diseases, it is necessary to comprehensively compare the safety of Dmab and ZA based on a wide range of bone-related diseases, which is also an important aspect to guide the clinical medication. Therefore, in this study, we conducted a systematic review and meta-analysis based on clinical trials to compare the safety and efficacy between Dmab and ZA in patients with bone-related diseases.

\section{Material and methods}

Registration of this systematic review has been completed on the PROSPERO (International Prospective Register of Systematic Reviews) website, under the registration number CRD42021227328. This systematic review was conducted with adherence to the guidelines of the Preferred Reporting Items for Systematic Reviews and Meta-analysis (PRISMA) statement [24].

\section{Study selections}

Relevant studies were searched and identified by individually searching the following databases: PubMed, Embase, Web of Science, the Cochrane Central Library, and ClinicalTrials.gov up to $1^{\text {st }}$ January 2021. For all databases, the following key terms were used for searching: "denosumab", "zoledronic acid" and "bone". The study design was limited to randomized controlled trials (RCTs). This meta-analysis adhered to the Critical Appraisal Skills Programme (CASP) Checklist. Eligibility assessment was performed by two independent reviewers (L.W.H. and J.R.Y.). Disagreements between reviewers were resolved by group discussion and consensus.

\section{Inclusion and exclusion criteria}

Eligibility was assessed by two independent reviewers (L.W.H. and J.R.Y.), with consensus reached by discussing conflicts with a third investigator (L.Y.). Assessments were performed and repeated twice. Only RCTs were included. First, the titles and abstracts were assessed. Full texts of potentially qualified studies were then obtained and carefully reviewed. Reviewers were not blinded to the authorship of the studies. Dissertations, conference proceedings, and studies in non-English languages were excluded.

\section{Outcomes of interest}

The primary outcome measure was the rate of adverse events. The secondary outcome measures were the rates of serious adverse events, SREs and infection events.

\section{Data collection}

The following data were extracted: first author, year of study, country of origin, study population, number of patients, basic demographic characteristics, treatment information and data of outcomes of interest. The data were extracted and cross-checked independently by two authors (L.W.H. and J.R.Y.). Disagreements were resolved through deep discussion with a third reviewer (L.Y.) until we reached a consensus.

\section{Evaluation of quality of evidence}

The methodological quality of the selected studies was blindly evaluated by two independent reviewers (L.W.H. and J.R.Y.). Disagreements were discussed among the group and resolved by a third assessor (L.Y.). The study quality was assessed using the CASP Checklist (Table I), which evaluates the risk of bias and comprises 11 items related to methodological quality and statistical reporting. Discrepancies and disagreements were resolved by consensus.

\section{Statistical analysis}

Data analyses were performed using the Cochrane Collaboration's Review Manager program (RevMan version 5.3; Cochrane Collaboration, 
Table I. Critical Appraisal Skills Programme (CASP) Checklist

\begin{tabular}{|lr|}
\hline Item number & Was the assigned treatment adequately concealed before allocation? \\
\hline 1 & Were the outcome of patients who withdrew described and included in the analysis (intention to treat)? \\
\hline 2 & Were the outcome assessors blinded to the treatment status? \\
\hline 3 & Were the treatment and control groups comparable at entry? \\
\hline 5 & Were the participants blinded to the assignment status after allocation? \\
\hline 6 & Were the treatment providers blind to the assignment status? \\
\hline 7 & Were the care programs, other than the trial options, identical? \\
\hline 8 & Were the inclusion and exclusion criteria clearly defined? \\
\hline 9 & Were the interventions clearly defined? \\
\hline 10 & Were the outcome measures used clearly defined? \\
\hline 11 & Were diagnostic tests used in the outcome assessment clinically useful? \\
\hline
\end{tabular}

Oxford, UK). Meta-analysis was conducted to calculate pooled odds ratios (ORs) with $95 \%$ confidence intervals ( $\mathrm{Cls}$ ). We evaluated heterogeneity across studies using the Cochrane chi-square $\left(\chi^{2}\right)$ test and quantified it with the $R^{2}$ statistic [25]. $I^{2}$ values of $25 \%, 50 \%$ and $75 \%$ represented low, moderate and high heterogeneity, respectively [26]. Fixed-effects or random-effects models were used accordingly. The publication bias was detected by funnel plots and was statistically examined by Egger's test [27]. Egger's test was performed in STATA version 16 (StataCorp, College Station, TX).

\section{Results}

\section{Literature search}

A flow diagram of the literature search is shown in Figure 1. Among 565 potentially eligible articles, 13 fulfilled the inclusion criteria. Initially, through the electronic database search, we identified 565 citations. Examinations of the reference lists in all relevant papers, recent editorials, and related review articles yielded no further studies for evaluation. Non-RCTs were excluded and the remaining 26 articles were then selected after reading the titles and abstracts. After reading the full texts, 13 studies were further excluded because they did not report relevant outcomes. The remaining 13 RCTs met our inclusion criteria and were ultimately included in the qualitative analysis and final meta-analysis.

\section{Study characteristics}

The characteristics of enrolled RCTs were presented in Table II. Our meta-analysis included 21,042 patients (10,073 men and 10,969 women) who were diagnosed with bone-related disease from six different countries. Among them, 10,535 (50.1\%) patients were treated with Dmab and 10,507 (49.9\%) patients were treated with ZA.

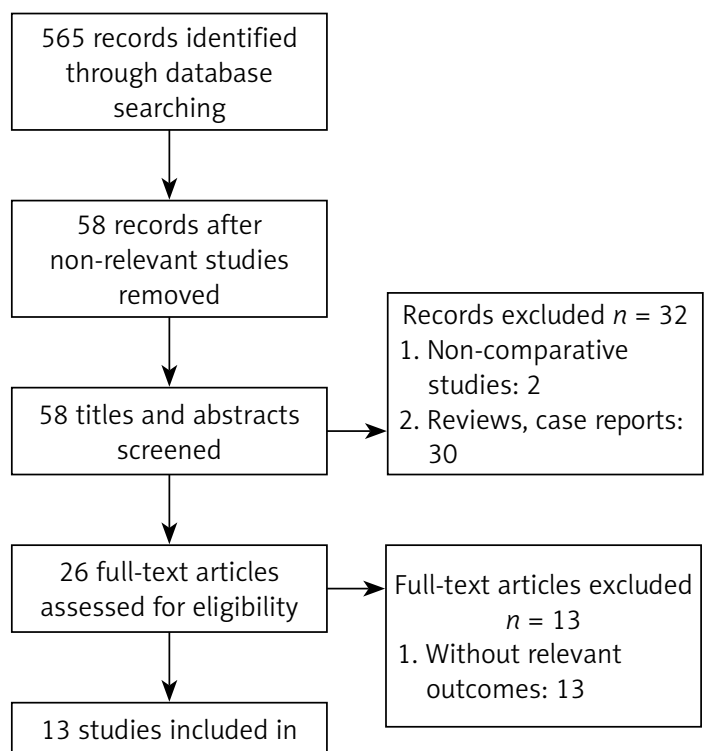

current meta-analysis

Figure 1. Flow diagram of the literature search

The results of the quality assessment of the included RCTs are detailed in Table III.

\section{Primary outcome}

\section{Adverse events}

Ten of the included studies reported the overall rate of adverse events. The adverse events rate was $86.3 \%(6581 / 7623)$ in the Dmab group and $87.6 \%(6644 / 7584)$ in the ZA group $(O R=0.84$, $95 \% \mathrm{Cl}: 0.75-0.94, p=0.003$ ) (Figure 2).

\section{Secondary outcomes}

\section{Serious adverse events}

Nine RCTs reported relevant data regarding the rate of serious adverse events. The incidence of serious adverse events was significantly lower in the Dmab group compared with the ZA group $(\mathrm{OR}=0.91,95 \% \mathrm{Cl}: 0.85-0.99, p=0.02)$ (Figure 3). 
Table II. Characteristics of randomized controlled trials enrolled in the meta-analysis

\begin{tabular}{|lcccccccc|}
\hline Year & Country & Design & \multicolumn{3}{c}{ D group } & \multicolumn{3}{c|}{ Z group } \\
\cline { 3 - 9 } & & $\begin{array}{c}\text { Patients } \\
\text { number }\end{array}$ & Median age & $\begin{array}{c}\text { Male } \\
\text { percentage }\end{array}$ & $\begin{array}{c}\text { Patients } \\
\text { number }\end{array}$ & Median age & $\begin{array}{c}\text { Male } \\
\text { percentage }\end{array}$ \\
\hline 2010 & America & RCT & 1026 & 57 & 0.8 & 1020 & 56 & 0.9 \\
\hline 2011 & America & RCT & 886 & 60 & 66.0 & 890 & 61 & 62.0 \\
\hline 2011 & France & RCT & 950 & 71 & 100.0 & 951 & 71 & 100.0 \\
\hline 2012 & Italy & RCT & 411 & 60 & 74.0 & 400 & 61 & 68.0 \\
\hline 2012 & America & RCT & 886 & 60 & 66.0 & 890 & 61 & 62.0 \\
\hline 2012 & Spain & RCT & 1026 & 57 & 0.8 & 1020 & 56 & 0.9 \\
\hline 2014 & America & RCT & 800 & 59 & 66.0 & 797 & 61 & 62.0 \\
\hline 2015 & France & RCT & 950 & 71 & 100.0 & 951 & 71 & 100.0 \\
\hline 2015 & Germany & RCT & 1912 & 58 & 31.0 & 1910 & 59 & 29.0 \\
\hline 2016 & America & RCT & 321 & 68.5 & 0.0 & 322 & 69.5 & 0.0 \\
\hline 2018 & America & RCT & 859 & 63 & 54.0 & 859 & 63 & 55.0 \\
\hline 2018 & Greece & RCT & 30 & 64.8 & 0.0 & 27 & 65.2 & 0.0 \\
\hline 2016 & America & RCT & 325 & 56 & 0.0 & 342 & 55.9 & 0.0 \\
\hline & & & 153 & 70 & 100.0 & 128 & 71 & 100.0 \\
\hline
\end{tabular}

Additions for Table II

\begin{tabular}{|lccccc|}
\hline References & Year & Country & Design & $\begin{array}{c}\text { D group } \\
\text { intervention }\end{array}$ & $\begin{array}{c}\text { Z group } \\
\text { intervention }\end{array}$ \\
\hline Stopeck, Alison T. & 2010 & America & RCT & $120 \mathrm{mg} \mathrm{q4w} \mathrm{s.c.}$ & $4 \mathrm{mg} \mathrm{q4w} \mathrm{ivgtt}$ \\
\hline Henry, David H. & 2011 & America & RCT & $120 \mathrm{mg} \mathrm{q4w} \mathrm{s.c.}$ & $4 \mathrm{mg} \mathrm{q4w} \mathrm{ivgtt}$ \\
\hline Fizazi, Karim & 2011 & France & RCT & $120 \mathrm{mg} \mathrm{q4w} \mathrm{s.c.}$ & $4 \mathrm{mg} \mathrm{q4w} \mathrm{ivgtt}$ \\
\hline Scagliotti, Giorgio Vittorio & 2012 & Italy & RCT & $120 \mathrm{mg} \mathrm{q4w} \mathrm{s.c.}$ & $4 \mathrm{mg} \mathrm{q4w} \mathrm{ivgtt}$ \\
\hline Vadhan-Raj, Saroj & 2012 & America & RCT & $120 \mathrm{mg} \mathrm{q4w} \mathrm{s.c.}$ & $4 \mathrm{mg} \mathrm{q4w} \mathrm{ivgtt}$ \\
\hline Martin, Miguel & 2012 & Spain & RCT & $120 \mathrm{mg} \mathrm{q4w} \mathrm{s.c.}$ & $4 \mathrm{mg} \mathrm{q4w} \mathrm{ivgtt}$ \\
\hline Henry, David & 2014 & America & RCT & $120 \mathrm{mg} \mathrm{q4w} \mathrm{s.c.}$ & $4 \mathrm{mg} \mathrm{q4w} \mathrm{ivgtt}$ \\
\hline Smith, Matthew R. & 2015 & France & RCT & $120 \mathrm{mg} \mathrm{q4w} \mathrm{s.c.}$ & $4 \mathrm{mg} \mathrm{q4w} \mathrm{ivgtt}$ \\
\hline Diel, Ingo J. & 2015 & Germany & RCT & $120 \mathrm{mg} \mathrm{q4w} \mathrm{s.c.}$ & $4 \mathrm{mg} \mathrm{q4w} \mathrm{ivgtt}$ \\
\hline Miller, Paul D. & 2016 & America & RCT & $60 \mathrm{mg} \mathrm{q6m} \mathrm{twice,} \mathrm{s.c.}$ & $5 \mathrm{mg}$ once ivgtt \\
\hline Raje, Noopur & 2018 & America & RCT & $120 \mathrm{mg} \mathrm{q4w} \mathrm{s.c.}$ & $4 \mathrm{mg} \mathrm{q4w} \mathrm{ivgtt}$ \\
\hline Anastasilakis, Athanasios D. & 2018 & Greece & RCT & $60 \mathrm{mg} \mathrm{q6m} \mathrm{twice,} \mathrm{s.c.}$ & $5 \mathrm{mg}$ once ivgtt \\
\hline Stopeck, Alison T. & 2016 & America & RCT & $120 \mathrm{mg} \mathrm{q4w} \mathrm{s.c.}$ & $4 \mathrm{mg} \mathrm{q4w} \mathrm{ivgtt}$ \\
\hline & & & & $120 \mathrm{mg} \mathrm{q4w} \mathrm{s.c.}$ & $4 \mathrm{mg} \mathrm{q4w} \mathrm{ivgtt}$ \\
\hline
\end{tabular}

\begin{tabular}{|lccc|}
\hline Year & Country & Design & Primary disease \\
\hline 2010 & America & RCT & Advanced breast cancer with bone metastases \\
\hline 2011 & America & RCT & Advanced cancer or multiple myeloma with bone metastases \\
\hline 2011 & France & RCT & Castration-resistant prostate cancer with bone metastases \\
\hline 2012 & Italy & RCT & Lung cancer with bone metastases \\
\hline 2012 & America & RCT & Advanced cancer or multiple myeloma with bone metastases \\
\hline 2012 & Spain & RCT & Advanced breast cancer \\
\hline 2014 & America & RCT & Advanced solid tumor with bone metastases \\
\hline 2015 & Grance & RCT & Castration-resistant prostate cancer with bone metastases \\
\hline 2016 & America & RCT & Advanced breast cancer and other solid tumors (excluding breast or prostate \\
cancer) or multiple myeloma with bone metastases
\end{tabular}




\section{Skeletal-related events}

The SRE rates were reported in four RCTs. The overall SRE rate was $40.5 \%$ (37.5\% in the Dmab group and $43.5 \%$ in the ZA group). Dmab contributed to a lower incidence of SRES (OR $=0.77$, 95\% Cl: 0.70-0.85, $p=0.00001$ ) (Figure 4).

\section{Infection events}

Four studies involving 6594 patients were pooled and analyzed. These four trials comparing Dmab with ZA in patients with bone-related disease showed no significant difference between the two drugs in the incidence of infection events $(\mathrm{OR}=1.06,95 \% \mathrm{Cl}: 0.93-1.20, p=0.39)$ (Figure 5).

\section{Publication bias}

Funnel plots for the incidence of adverse events, serious adverse events, infection events and SREs are presented in Figure 6. The funnel plots did not show obvious asymmetry, and only one study (Fizazi, Karim 2011 [7]) evaluating the incidence of serious adverse events lay outside the limits of the $95 \% \mathrm{Cl}$. Considering that the accuracy of funnel plots might be limited by the small number of studies, we complemented them with Egger's test to statistically examine the publication bias. Egger's test suggested no significant publication bias for the incidence of adverse events $(p=0.310)$, serious adverse events $(p=0.713)$, infection events $(p=0.388)$ or SREs $(p=0.554)$.

\section{Discussion}

We obtained several major findings from the present meta-analysis based on data from 21,042 patients with bone-related diseases. From an efficacy perspective, Dmab resulted in fewer SREs in patients with bone metastases compared with ZA. For medication safety, Dmab significantly reduced the overall rate of adverse events including severe adverse events compared with ZA. Moreover, Dmab did not induce a higher risk of infection.

The benefit of preventing SREs in patients with bone metastases achieved by Dmab was consistently reported across included clinical trials with no interstudy heterogeneity. Previous metaanalyses have also confirmed the advantage of Dmab over ZA in delaying the onset of SREs [2123]. SREs secondary to bone metastases such as pathological fracture, spinal cord compression, radiation or surgery to bone commonly occur clinically [28], resulting in reduced survival, higher functional independence rates and dramatically lower health-related quality of life [29]. Moreover, SREs impose a considerable financial burden on patients due to subsequent treatments [30, 31]. Although the direct drug cost for Dmab was higher

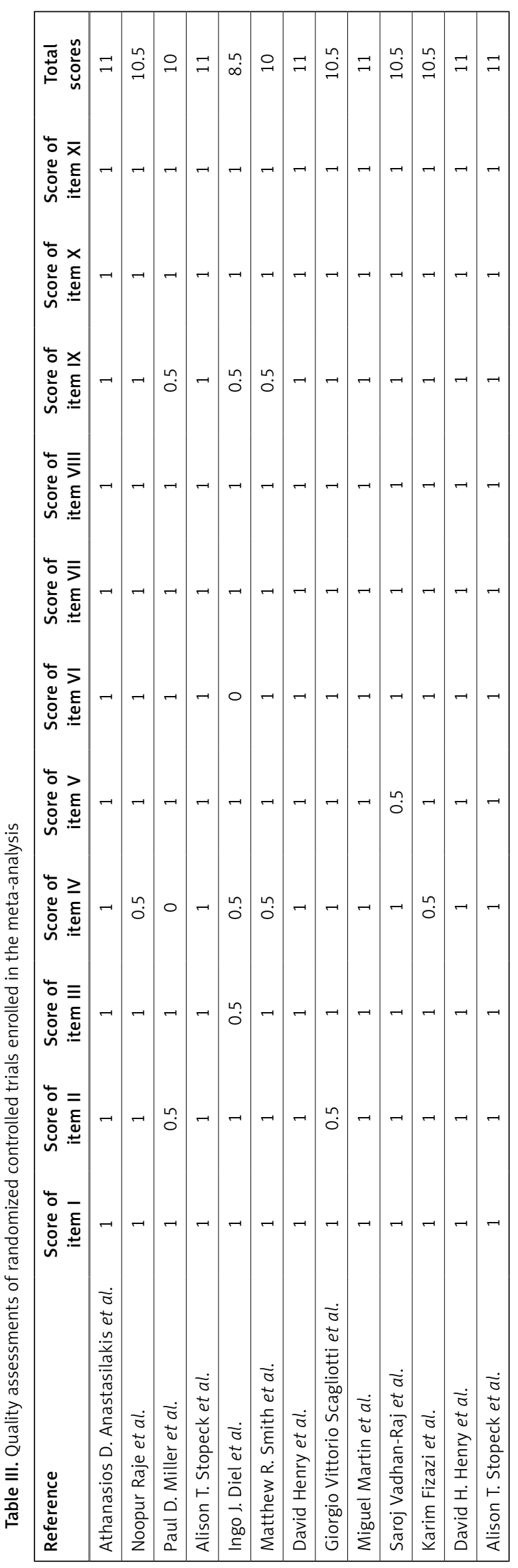




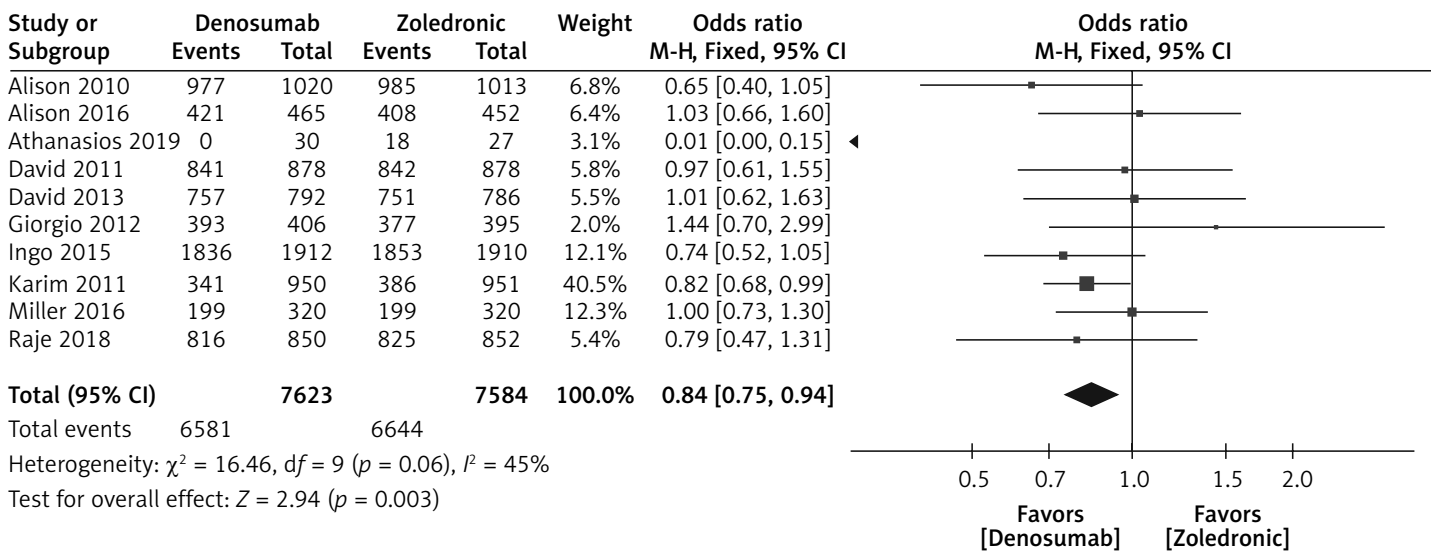

Figure 2. Forest plot for the incidence of adverse events in denosumab compared with zoledronic acid

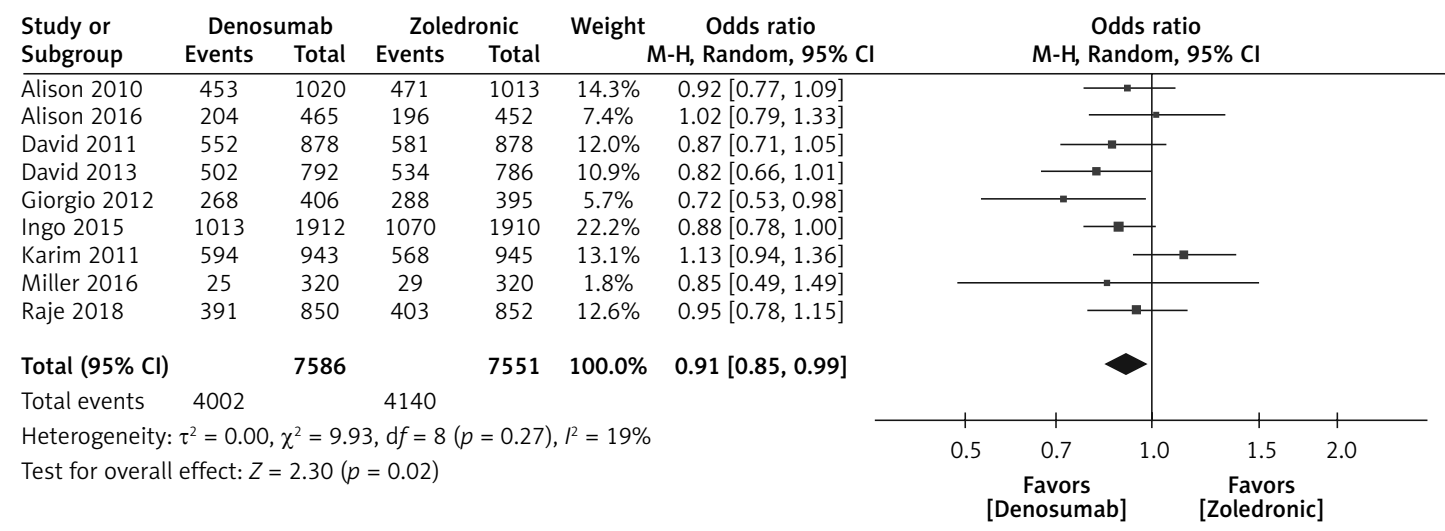

Figure 3. Forest plot for the incidence of serious adverse events in denosumab compared with zoledronic acid

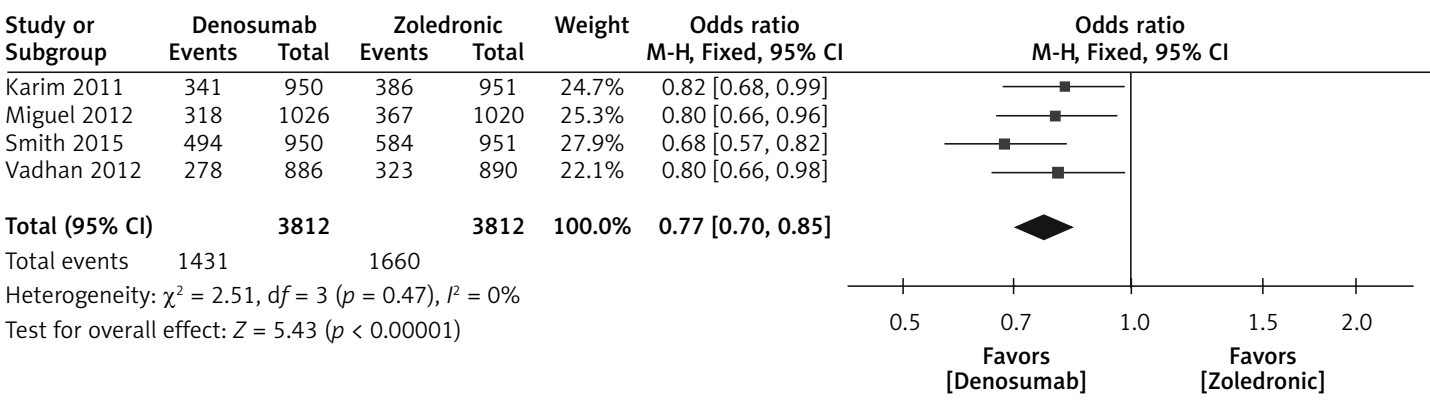

Figure 4. Forest plot for the incidence of SREs in denosumab compared with zoledronic acid

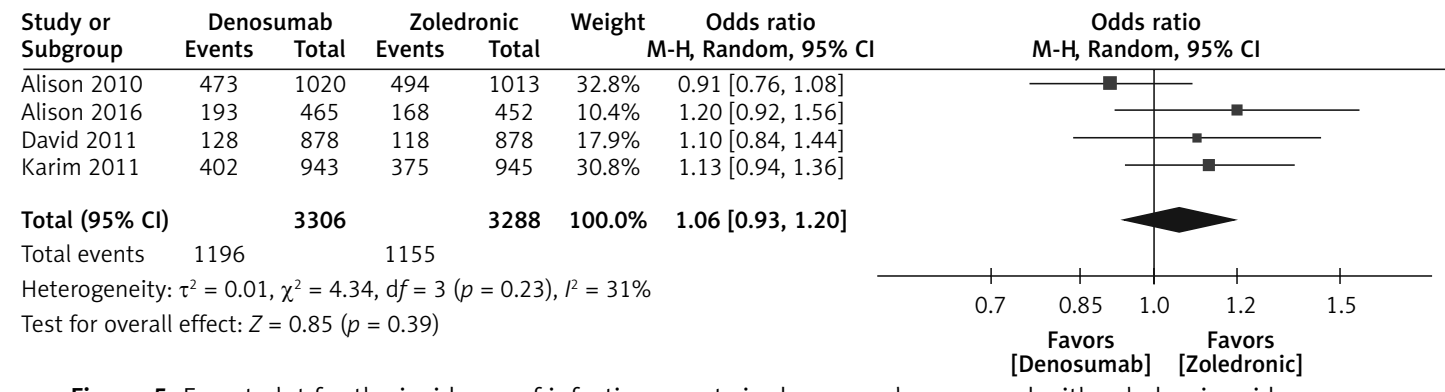

Figure 5. Forest plot for the incidence of infection events in denosumab compared with zoledronic acid 
A

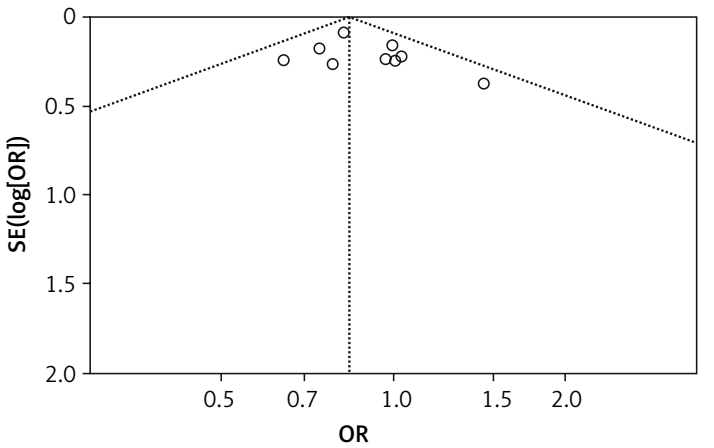

C

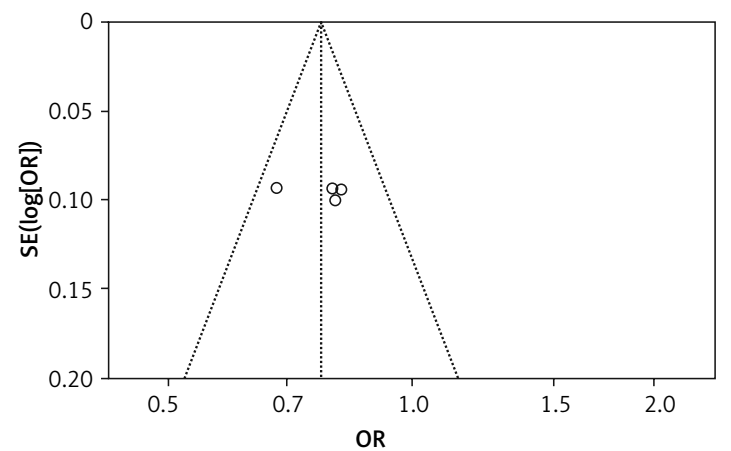

B

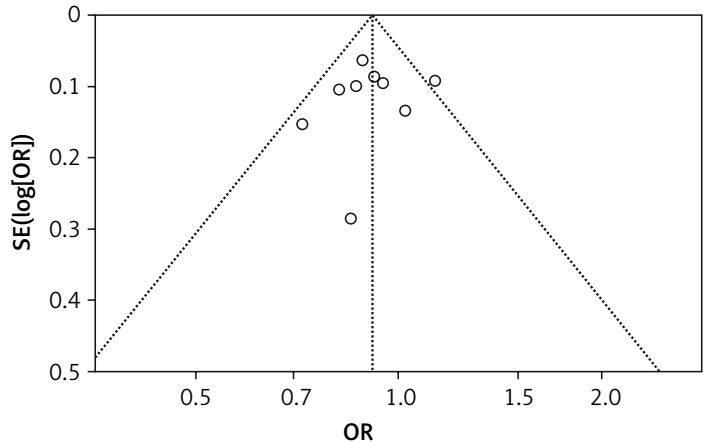

D

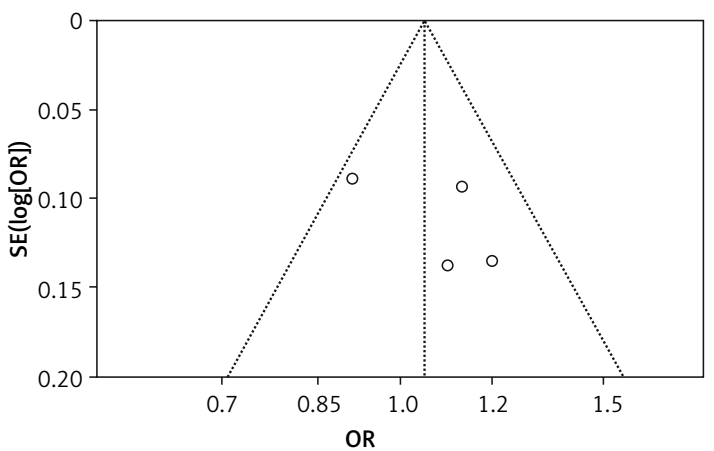

Figure 6. Funnel plots for the incidence of: $\mathbf{A}$ - adverse events, $\mathbf{B}$ - serious adverse events, $\mathbf{C}$ - infection events and D - skeletal-related events

than ZA, it can be remarkably offset by reduced costs contributed by preventing or delaying the onset of SREs $[19,20]$. Therefore, compared with ZA, Dmab can alleviate both the health and economic burden for patients.

The comparison of the overall adverse events rate between Dmab and ZA has been little evaluated in previous meta-analyses. After processing data from ten RCTs which enrolled a total of 15,207 patients, our analyses indicated that Dmab was superior to $Z A$ in reducing the overall rate of adverse events. Of the ten studies, three included patients with multiple myeloma $[8,10,32]$ and two included patients with postmenopausal osteoporosis $[5,18]$, which relatively well represented the spectrum of indications of antiresorptive regents. Of note, no adverse events were recorded in the Dmab group in one study based on patients with postmenopausal osteoporosis [5], which was also the major source of heterogeneity. One potential explanation was that all patients underwent previous treatment of Dmab with a mean duration of 2.2 years before the start of the trial and thus well tolerated a second course of Dmab treatment. After excluding this study for sensitivity analysis, the result remained significant with a remarkable decrease in heterogeneity $\left(p=0.020, l^{2}=0\right)$. Moreover, Dmab was also associated with fewer serious adverse events after evaluating data from nine clinical trials. A previous meta-analysis based on patients with bone metastases demonstrated that Dmab administration was associated with lower risk of serious adverse events including hypocalcemia, new primary malignancy and particularly renal toxicity [16], which together with the results of our meta-analysis confirmed that Dmab had advantages in reducing the occurrence of serious adverse events over ZA.

The RANKL pathway is expressed in activated lymphocytes and is involved in the formation of lymphoid nodes and the thymic microenvironment $[33,34]$, and its inhibition by Dmab was found to be correlated with a higher risk of infection. As shown by the results of our analysis, Dmab did not significantly increase the incidence of infection events compared with ZA. However, according to the pooled estimate of four included clinical trials, the overall rate of infection after infusion of Dmab was $36.2 \%$. Additionally, serious and opportunistic infections have been observed, though rarely, in patients treated with Dmab [35, 36]. Therefore, Dmab-induced infection still merits consideration before the initiation of therapy.

The present meta-analysis provided an assessment of current evidence regarding the efficacy and safety of Dmab versus ZA based on 13 high-quality RCTs which covered several bone-related diseases. To our current knowledge, compared with previous studies regarding the related topic, this meta-analysis contains the largest 
number of RCTs and covers the widest range of bone-related diseases, contributing to a reliable result and a more extensive application of analysis results. Despite these strengths, our study has several limitations. Even though the studies included in our meta-analysis were not confined to bone metastases, the number of studies evaluating non-cancer diseases such as postmenopausal osteoporosis was too small to conduct a reliable and robust subgroup analysis, which may limit the generalization of our results. For osteoporosis, the results must be interpreted with caution, and a subgroup analysis is warranted with more articles published. Also, some included studies were sponsored by pharmaceutical companies, and as such they were not free of potential pharmaceutical company bias.

\section{Conclusions}

Based on 13 high-quality randomized clinical trials, our results demonstrated that Dmab was superior to ZA in reducing the overall rate of adverse events as well as serious adverse events, and in reducing the onset of SREs. The treatment of denosumab was not correlated with a higher risk of infection as previously found. Considering the superiority of denosumab in safety outcomes, denosumab will be regarded as an optimal intervention for bone-related diseases. However, for bone-related diseases other than bone metastases, the superior safety of denosumab should be generalized with caution and further analyses are still warranted.

\section{Conflict of interest}

The authors declare no conflict of interest.

\section{References}

1. Duman S, Çamurcu iY, Uçpunar H, Sevencan A, Akıncı Ş, Sahin V, et al. Comparison of clinical characteristics and 10-year survival rates of revision hip arthroplasties among revision time groups. Arch Med Sci 2021; 17: 382-9.

2. Xu N, Wang L, Fu S, Jiang B. Resveratrol is cytotoxic and acts synergistically with NF- $\mathrm{KB}$ inhibition in osteosarcoma MG-63 cells. Arch Med Sci 2021; 17: 166-76.

3. Yin $\mathrm{P}$, Shi Q, Xiao F, et al. Inhibition of miR-22 promotes differentiation of osteoblasts and improves bone formation via the YWHAZ pathway in experimental mice. Arch Med Sci 2020; 16: 1419-31.

4. Sert IU, Kilic O, Akand M, Saglik L, Avunduk MC, Erdemli E. The role of vitamin $E$ in the prevention of zoledronic acid-induced nephrotoxicity in rats: a light and electron microscopy study. Arch Med Sci 2018; 14: 381-7.

5. Anastasilakis AD, Papapoulos SE, Polyzos SA, Appelman-Dijkstra NM, Makras P. Zoledronate for the prevention of bone loss in women discontinuing denosumab treatment. a prospective 2-year clinical trial. J Bone Miner Res 2019; 34: 2220-8.
6. Cummings SR, San Martin J, McClung MR, et al. Denosumab for prevention of fractures in postmenopausal women with osteoporosis. N Engl J Med 2009; 361: 756-65.

7. Fizazi K, Carducci $M$, Smith $M$, et al. Denosumab versus zoledronic acid for treatment of bone metastases in men with castration-resistant prostate cancer: a randomised, double-blind study. Lancet 2011; 377: 813-22.

8. Henry DH, Costa L, Goldwasser F, et al. Randomized, double-blind study of denosumab versus zoledronic acid in the treatment of bone metastases in patients with advanced cancer (excluding breast and prostate cancer) or multiple myeloma. J Clin Oncol 2011; 29: 1125-32.

9. Lipton A, Fizazi K, Stopeck AT, et al. Effect of denosumab versus zoledronic acid in preventing skeletal-related events in patients with bone metastases by baseline characteristics. Eur J Cancer 2016; 53: 75-83.

10. Raje N, Terpos E, Willenbacher W, et al. Denosumab versus zoledronic acid in bone disease treatment of newly diagnosed multiple myeloma: an international, double-blind, double-dummy, randomised, controlled, phase 3 study. Lancet Oncol 2018; 19: 370-81.

11. Raje N, Vadhan-Raj S, Willenbacher W, et al. Evaluating results from the multiple myeloma patient subset treated with denosumab or zoledronic acid in a randomized phase 3 trial. Blood Cancer J 2016; 6: e378.

12. Lipplaa A, Kroep JR, van der Heijden L, et al. Adjuvant zoledronic acid in high-risk giant cell tumor of bone: a multicenter randomized phase II trial. Oncologist 2019; 24: 889-e421.

13. van der Heijden L, Dijkstra PDS, Blay JY, Gelderblom H. Giant cell tumour of bone in the denosumab era. Eur J Cancer 2017; 77: 75-83.

14. Kohno N, Aogi K, Minami H, et al. Zoledronic acid significantly reduces skeletal complications compared with placebo in Japanese women with bone metastases from breast cancer: a randomized, placebo-controlled trial. J Clin Oncol 2005; 23: 3314-21.

15. Body JJ, Facon T, Coleman RE, et al. A study of the biolog ical receptor activator of nuclear factor-kappaB ligand inhibitor, denosumab, in patients with multiple myeloma or bone metastases from breast cancer. Clin Cancer Res 2006; 12: 1221-8.

16. Chen F, Pu F. Safety of denosumab versus zoledronic acid in patients with bone metastases: a meta-analysis of randomized controlled trials. Oncol Res Treat 2016; 39: 453-9.

17. Scagliotti GV, Hirsh V, Siena S, et al. Overall survival improvement in patients with lung cancer and bone metastases treated with denosumab versus zoledronic acid: subgroup analysis from a randomized phase 3 study. J Thorac Oncol 2012; 7: 1823-9.

18. Miller PD, Pannacciulli N, Brown JP, et al. Denosumab or zoledronic acid in postmenopausal women with osteoporosis previously treated with oral bisphosphonates. J Clin Endocrinol Metab 2016; 101: 3163-70.

19. Stopeck A, Brufsky A, Kennedy L, et al. Cost-effectiveness of denosumab for the prevention of skeletal-related events in patients with solid tumors and bone metastases in the United States. J Med Econ 2020; 23: 37-47.

20. Raje N, Roodman GD, Willenbacher W, et al. A cost-effectiveness analysis of denosumab for the prevention of skeletal-related events in patients with multiple myeloma in the United States of America. J Med Econ 2018; 21: 525-36.

21. Sun L, Yu S. Efficacy and safety of denosumab versus zoledronic acid in patients with bone metastases: 
a systematic review and meta-analysis. Am J Clin Oncol 2013; 36: 399-403.

22. Chen C, Li R, Yang T, et al. Denosumab versus zoledronic acid in the prevention of skeletal-related events in vulnerable cancer patients: a meta-analysis of randomized, controlled trials. Clin Ther 2020; 42: 1494-507.e1.

23. Chen J, Zhou L, Liu X, Wen X, Li H, Li W. Meta-analysis of clinical trials to assess denosumab over zoledronic acid in bone metastasis. Int J Clin Pharm 2021; 43: 2-10.

24. Moher D, Liberati A, Tetzlaff J, Altman DG, PRISMA Group. Preferred reporting items for systematic reviews and meta-analyses: the PRISMA statement. Ann Intern Med 2009; 151: 264-9, W64.

25. Higgins JP, Thompson SG. Quantifying heterogeneity in a meta-analysis. Stat Med 2002; 21: 1539-58.

26. Higgins JP, Thompson SG, Deeks JJ, Altman DG. Measuring inconsistency in meta-analyses. BMJ 2003; 327: 557-60.

27. Egger M, Davey Smith G, Schneider M, Minder C. Bias in meta-analysis detected by a simple, graphical test. BMJ 1997; 315: 629-34.

28. D’Oronzo S, Coleman R, Brown J, Silvestris F. Metastatic bone disease: Pathogenesis and therapeutic options: Up-date on bone metastasis management. J Bone Oncol 2019; 15: 004-4.

29. Costa L, Badia X, Chow E, Lipton A, Wardley A. Impact of skeletal complications on patients' quality of life, mobility, and functional independence. Support Care Cancer 2008; 16: 879-89.

30. Yong C, Onukwugha E, Mullins CD. Clinical and economic burden of bone metastasis and skeletal-related events in prostate cancer. Curr Opin Oncol 2014; 26: 274-83.

31. Bhowmik D, Hines DM, Intorcia M, Wade RL. Economic burden of skeletal-related events in patients with multiple myeloma: analysis of US commercial claims database. J Med Econ 2018; 21: 622-8.

32. Diel IJ, Body JJ, Stopeck AT, et al. The role of denosum$a b$ in the prevention of hypercalcaemia of malignancy in cancer patients with metastatic bone disease. Eur J Cancer 2015; 51: 1467-75.

33. Leibbrandt A, Penninger JM. RANK/RANKL: regulators of immune responses and bone physiology. Ann N Y Acad Sci 2008; 1143: 123-50.

34. Dore RK. The RANKL pathway and denosumab. Rheum Dis Clin North Am 2011; 37: 433-52, vi-vii.

35. Watts NB, Roux C, Modlin JF, et al. Infections in postmenopausal women with osteoporosis treated with denosumab or placebo: coincidence or causal association? Osteoporosos Int 2012; 23: 327-37.

36. Lau AN, Wong-Pack M, Rodjanapiches R, et al. Occurrence of serious infection in patients with rheumatoid arthritis treated with biologics and denosumab observed in a clinical setting. J Rheumatol 2018; 45: 170-6. 